Article

\title{
Propagation and transformation of vortexes in linear and non- linear radio-photon systems
}

\author{
Valery H. Bagmanov 1, Albert Kh. Sultanov ${ }^{1}$, Ivan K. Meshkov ${ }^{1}$, Azat R. Gizatulin ${ }^{1}$, \\ Raoul R. Nigmatullin ${ }^{2 *}$, Airat Zh. Sakhabutdinov ${ }^{3}$
}

1 Ufa State Aviation Technical University; bagmanov.valeriy@yandex.ru (V.H.B.); sultanov.ah@mail.ru (A.Kh.S.); mik.ivan@bk.ru (I.K.M.); azat_poincare@mail.ru (A.R.G.);

2 Department of Radio-Electronic and Information-Measuring Tools, Kazan National Research Technical University Named after A.N. Tupolev-KAI, K. Marx Str. 10, 420111 Kazan, Russia; renigmat@gmail.com (R.R.N.);

3 Department of Radiophotonics and Microwave Technologies, Kazan National Research Technical University Named after A.N. Tupolev-KAI, K. Marx Str. 10, 420111 Kazan, Russia; azhsakhabutdinov@kai.ru (A.Zh.S.);

* Correspondence: renigmat@gmail.com (R.R.N.)

\begin{abstract}
The article is devoted to issues related to the propagation and transformation of vortexes in the optical range of frequency. Within the framework of the traditional and modified model of slowly varying envelope approximation (SVEA), the process of converting vortex beams of the optical domain into vortex beams of the terahertz radio range based on nonlinear generation of a difference frequency in a medium with a second-order susceptibility is considered. The modified SVEA splits a slowly varying amplitude into two factors, which makes it possible to more accurately describe the three-wave mixing process. The theoretical substantiation of the rule of vortex beams topological charges conversion is given - the topological charge of the output radio-vortex beam is equal to the difference between the topological charges of the input optical vortex beams. A numerical simulation model of the processes under consideration has been implemented and analyzed.
\end{abstract}

Keywords: vortex propagation, difference frequency generation, nonlinear medium, vortex beams conversion.

\section{Introduction}

In recent decades in the field of telecommunications there has been an increased interest in the use of vortex beams - electromagnetic waves with orbital angular momentum (OAM) - a special type of wavefront with helicoidal dislocation, characterized by "donut" shape of intensity pattern and phase singularity in the center of the field distribution. This interest is due to the fact that various quantized states of the projection of the orbital angular momentum on the direction of propagation can be used both for coding signals and for multiplexing of communication channels. Thus, vortex beams open up additional opportunities both for increasing the information transfer rate (capacity) and for increasing the noise immunity and security of communication channels since the OAM order of wave can be decoded only by coaxial receiving (line of sight). One of the main directions in the development of infocommunication technologies is radiophotonics - hybrid systems that combine the processes of signals processing, transfer and conversion in both radio and optical ranges. At present, in radiophotonics, the microwave range is being investigated, including the terahertz frequency range of $0.1-50 \mathrm{THz}$ as the most promising from the point of view of low congestion of the electromagnetic spectrum. Moreover, such a range promises higher data transfer rates as well.

A promising direction of radiophotonics in the field of telecommunications is the development of Radio-over-fiber (RoF) systems [1], which use the transmission of mi- 
crowave radio signals over optical fiber without need of $\mathrm{GHz}$ local oscillator (LO) at the remote antenna unit (RAU), which makes it possible to directly convert modulated optical signal into radio domain (after amplification and filtering) and transmit it in air channel. In the context of the development of RoF systems, the problem arises of converting signals that carry orbital angular momentum in the optical domain into signals that carry OAM in the radio range. In this paper, we consider the process of converting vortex beams of the optical range in the wavelength range $\lambda \approx 1.5 \mu \mathrm{m}$ (C-band) into vortex beams of the radio band in the range of frequencies exceeding $100 \mathrm{GHz}$.

Nonlinear media with nonzero polarizability (dielectric susceptibility) of the second-order are widely used for converting electromagnetic waves from the optical domain into radio band in the terahertz range based on the difference frequency generation (DFG), second harmonic generation (SHG), etc. In this case, the nonlinear medium, which is the source of generation, is modified by additional inclusions (is doped) or structurally changed in order to increase the conversion efficiency. So, in [2], a lattice of photonic crystals is used do achieve effective DFG. In article [3], lasing occurs in a strip metal waveguide with the inclusion of GaAs impurities. In [4], a rectangular metal waveguide is used, filled with periodically alternating layers of $\mathrm{LiTaO}_{3}$ with the opposite orientation of the optical axes. Authors of [5] demonstrate the difference frequency generation in a 2D plasmon waveguide consisting of a $\mathrm{LiNbO}_{3}$ layer and located between two flat nonmetallic layers. In [6], the generation of a difference frequency by Gaussian beams is described, and the results of experimental generation of a difference frequency in $\mathrm{a} \mathrm{LiNbO}_{3}$ crystal containing planar waveguides are presented.

With regard to research on the transformation of vortex beams in nonlinear media with second-order polarizability, the following papers can be noted. It was shown in [7] that when the second harmonic is generated, the topological charge of the vortex beam $l$ doubles, where $l$ is an integer quantum number and determines the projection of the orbital angular momentum on the direction of propagation, due to the dependence of the electromagnetic field of the vortex beam on the azimuthal angle $\varphi$ in the form of $\mathrm{e}^{i l \varphi}$.

In [8], a hypothesis is expressed and an indirect theoretical substantiation and numerical simulation results are presented, confirming the fact that the topological charge of the output vortex beam is equal to the sum of the charges of the input vortex beams when generating the sum harmonic (frequency). In [9], the laws of conservation of the topological charge during the generation of the second harmonic, including the state with a fractional topological charge $l$, are studied theoretically and experimentally. The works $[10,11]$ experimentally confirm the law of conservation of the topological charge during the generation of the second harmonic [10] of the sum harmonic [11], where the theoretical substantiation of the results obtained is also given. In [12], it was experimentally established that in a multilayer nonlinear medium with periodically alternating layers of $\mathrm{LiTaO}_{3}$ with oppositely directed optical axes to ensure spatial phase matching during the generation of the total harmonic, topological charges are summed. In [13] a two-dimensional periodically poled $\mathrm{LiTaO}_{3}$ nonlinear photonic crystal is used to generate conical third harmonic; phase matching is achieved by crystal poling. Moreover, it was shown [14] that the filamentation of femtosecond pulses (used to ensure phase matching condition) allows the formation of a vortex mode due to the third-harmonic generation (THG) process in Ti: sapphire chirped pulse amplifier. Note that in this work, the law of conservation of the topological charge of vortex beams was justified theoretically; it was experimentally confirmed in [15] in the framework of the THG process in PPLT in the optical range: the processes of frequency doubling and generation of the total harmonic are considered. It is also shown in the article that the conversion efficiency decreases with an increase of the OAM order. The authors of [16] experimentally demonstrate the formation of multiple copies of the SH beams carrying the doubled OAMs using a two-dimensional PPLT crystal: the doubling of the topological charge during the second harmonic generation (SHG) in the vicinity of a wavelength of $920 \mathrm{~nm}$ is proved. Article [17] experimentally shows the possibility of generating the second harmonic by plas- 
mons, and the topological charge doubles as the frequency is doubled. PPLN crystals are also used to generate infrared radiation with a wavelength of the order of several microns [18]. In addition to PPLN / PPLT crystals, KTP crystals can also be used for SHG of Laguerre - Gauss modes [19]. In [20], a hybrid lithium niobate/silicon waveguide scheme with a high generation efficiency of the order of $3.5 \times 10^{-4} \mathrm{~W}^{-1}$ at $3 \mathrm{THz}$ is used to achieve a terahertz signal by generating a difference frequency. In addition to classical PPLNs, aperiodic PPLNs (APPLNs) are also investigated: in [21], a similar crystal is used for cascade DFG (CDFG), which results in terahertz radiation; the conversion efficiency of this approach is almost 3 times higher, but it is achieved at a low temperature - about $10 \mathrm{~K}$. In [22], the optical pump wave and the incident wave propagate in a lithium niobate crystal not co-directionally, but towards each other, which, according to the authors, allows optical feedback without resonance cavity and helps to generate narrow-band $\mathrm{THz}$ radiation. A terahertz signal can also be generated using a 2D InxGa1-xSe crystal [23] using DFG with a conversion efficiency of the order of $10^{-5}$.

Authors of [24] use computational experiments and theoretical description and models to show the influence of the length of a nonlinear medium on the process of parametric amplification of light in the process of Laguerre-Gaussian modes coupling, the law of conservation of topological charge is established, and the influence of the radial indices of Laguerre-Gaussian modes on the process of parametric amplification is also investigated. In [25], a theoretical model for nonlinear transformations of LG modes is developed: an exact solution for the single-pass no-cavity regime was obtained and simulation modeling was carried out. The authors of [26] obtained an analytical solution for terahertz waves in planar waveguides based on the coupled mode theory based on the DFG process.

Non-linear media can also be used in quantum applications. For example, to generate pairs of entangled photons carrying OAM. Thus, in [27], such photons are formed due to the spontaneous parametric down-conversion process in nonlinear 3-mm-long $\beta$-barium borate (BBO) crystal. It is shown that entangled photons conserve the orbital angular momentum in similar schemes used to test Bell's theorem. The authors of [28] use spontaneous four-wave mixing (SFWM) to create correlated photon pairs carrying OAM. These pairs can significantly increase the information capacity of quantum systems due to the additional physical degree of freedom; within the framework of [2], similar photonic systems were used to develop quantum memory based on cold Rb ensembles.

There are a lot of papers regarding the generation of terahertz vortices. For example, the authors of $[29,30]$ form an OAM signal in the $\mathrm{THz}$ range based on DFG (pump wavelengths $\lambda_{1}=1.56 \mu \mathrm{m}$ and $\left.\lambda_{2}=1.50 \sim 1.64 \mu \mathrm{m}\right) \quad$ using a 4'-dimethylamino$\mathrm{N}$-methyl-4-stilbazolium tosylate (DAST) crystal with fairly high efficiency (according to the authors, more than 90\%); however, only continuous wave systems are considered. In [31], terahertz vortex pulses of the order of 2 and -2 are also formed by DFG in ZnTe crystal with a pump wavelength of about $800 \mathrm{~nm}$. The conversion efficiency for $\mathrm{THz}$ signal generation using DFG can be increased by using nanophotonic lithium niobate chips or nanophotonic waveguides, as shown in [32] and [33], respectively. In addition to crystals, there are some interesting methods for generating $\mathrm{THz}$ vortexes using undulators [34]. It should be noted that the propagation of terahertz vortexes in the air [35] and optical fibers [36] is also currently being studied.

Finally, we can mention some alternative solutions for the $\mathrm{THz}$ generation. The terahertz signal in [37] is formed by fast tuning of the DFB laser optical frequency using a fiber optical interferometer. In [38], a terahertz signal with a power of the milliwatt order is nonlinearly generated using an optimized mid-infrared quantum cascade laser. The authors of [39] used intra-cavity mixing of two lasers - DFB and Fabry-Perot - operating in the infrared range to generate a frequency comb in the terahertz band with an input power of the order of several microwatts. In conclusion, we note that the conversion of OAM signals can also be implemented in a linear regime. For example, in [40], a theo- 
retical model of the linear transformation of infrared vortexes into the visible region was developed, and experimental confirmation of this process was carried out.

Analysis of works in the field of converting optical radiation into the radio range based on the use of nonlinear media with a second-order susceptibility shows that the theoretical substantiation and modeling of the ongoing generation processes is based on a system of three equations describing the processes within the slowly varying envelope approximation [41], regardless of the structural organization of a nonlinear environment.

Despite the "genetic" relationship of various three-wave mixing processes - generation of the second harmonic, sum frequency, parametric amplification, generation of a difference frequency - each of these processes has its own characteristics, in particular, a distinctive feature of the process of generating a difference frequency in the radio range is a very low energy conversion efficiency caused by two factors: 1 ) the smallness of the ratio of the nonlinear part of the polarization to the linear one, which is typical for all three-wave processes, excluding the region of extremely high values of the electric field strength of the initial harmonics; 2) due to the dipole mechanism of wave emission in the crystal, the power of the dipole radiation is proportional to the square of the frequency, therefore, in the investigated frequency range, the ratio of the radiation powers in both radio and optical ranges is a small quantity proportional to the square of the ratio of the corresponding frequencies and in the context of this work amounts to $\sim 10^{-5}-10^{-6}$ since the frequency of optical signal is about $200 \mathrm{THz}$ and the radio signal is only $0.1 \mathrm{THz}$.

The purpose of this work is to study the process of converting radiation from the optical range to the radio domain under the conditions specified previous paragraph.

\section{Transformation of vortex compositions in linear media}

In homogeneous linear media, electromagnetic waves are described by a system of inhomogeneous wave equations that are in fact a direct consequence of Maxwell's equations:

$$
\begin{aligned}
& \nabla^{2} \vec{A}-\frac{n^{2}}{c^{2}} \frac{\partial^{2} \bar{A}}{\partial t^{2}}=-\frac{4 \pi}{c} \vec{j} \\
& \nabla^{2} \varphi-\frac{n^{2}}{c^{2}} \frac{\partial^{2} \varphi}{\partial t^{2}}=4 \pi \rho
\end{aligned}
$$

where $c$ is the speed of light, $n$ is the refractive index, $\vec{A}$ is the vector potential, $\varphi$ is the scalar potential, $\vec{j}$ is the current density, $\rho$ is the density of free charges, $\nabla^{2}$ is the Laplace operator (in CGS system of units).

The emission of monochromatic waves with frequency $\omega$ is determined by the Helmholtz equations following from the system of equations (1).

$$
\begin{aligned}
& \left(\nabla^{2}+k^{2}\right) \vec{A}(\vec{r}, \omega)=-\frac{4 \pi}{c} \vec{j}(\vec{r}, \omega) \\
& \left(\nabla^{2}+k^{2}\right) \varphi(\vec{r}, \omega)=4 \pi \rho(\vec{r}, \omega)
\end{aligned}
$$

where $k=\frac{\omega n}{c}$ is the wave number and $\vec{A}(\vec{r}, \omega), \vec{j}(\vec{r}, \omega), \varphi(\vec{r}, \omega), \rho(\vec{r}, \omega)$ are the Fourier transforms of the corresponding fields.

From a mathematical point of view, the system of equations (2) is structurally equivalent to the inhomogeneous equation

$$
\left(\nabla^{2}-k^{2}\right) U(\vec{r})=f(\vec{r})
$$

where $U(r)$ is a certain radiated field (or one of the components in the case of a vector field), and $f(\vec{r})$ is a volumetric source of radiation.

In the most general approach, the problem of wave propagation in a linear medium can be formulated as a problem in which radiation is generated not only by a volumetric 
source $f(\vec{r})$, but also by some surface source $\psi$. Thus, the task is to find a solution of Eq. (3) in a half-space $z \geq 0$ of three-dimensional space $(x, y, z)$ if a certain initial field distribution $U(r)$ is given on the boundary plane $z=0$.

$$
\left.U(r)\right|_{z=0}=\psi(x, y)
$$

It is known that electromagnetic fields carrying angular momentum (vortex beams, vortexes) are spatial structures with a coordinate dependence in the Cartesian system given by

$$
U=(x+i y)^{m}
$$

or in a cylindrical coordinate system

$$
U=\rho e^{i m \varphi}
$$

where $m$ is the topological charge of the vortex, the physical meaning of which is that $m$ is proportional to the projection of the angular momentum on the direction of wave propagation (in this case $-z$ axis), and functions (5) or (6) are eigenfunctions of the $z$-component of the angular momentum operator and $i$ is imaginary one.

Let us show that a necessary condition for radiation carrying angular momentum in the case of continuous sources is the vortex structure of both volumetric and surface sources, which is preserved during propagation in space.

Now we consider equation (3) in the paraxial approximation. In this case, instead of equation (3), we will have the inhomogeneous parabolic equation

$$
\nabla_{\perp}^{2} U+2 i k \frac{\partial V}{\partial z}=f(x, y, z)
$$

where $\nabla_{\perp}^{2}=\frac{\partial^{2}}{\partial x^{2}}+\frac{\partial^{2}}{\partial y^{2}}$ is the transverse component of the Laplace operator. The solution to the radiation equation (7) and (4) is represented by the integral relation:

$$
\begin{aligned}
& U(x, y, z)=\int_{-\infty}^{+\infty} \int_{+\infty}^{+\infty} G\left(x-x^{\prime}, y-y^{\prime}, z\right) \psi\left(x^{\prime}, y^{\prime}\right) d x^{\prime} d y^{\prime}+ \\
& \int_{0}^{z} \int_{-\infty}^{+\infty} \int_{-\infty}^{+\infty} G\left(x-x^{\prime}, y-y^{\prime}, z-z^{\prime}\right) f\left(x^{\prime}, y^{\prime}, z^{\prime}\right) d x^{\prime} d y^{\prime} d z^{\prime}
\end{aligned}
$$

where the Green's function $G(x, y, z)$ has the Cartesian form

$$
G(x, y, z)=-\frac{1}{4 \pi z} \exp \left\{-i \frac{\left(x^{2}+y^{2}\right) k}{2 z}\right\}
$$

Now let's go over to a cylindrical coordinate system $(\rho, \varphi, z)$ and assume that both volumetric and surface sources have a vortex structure. In this case, the corresponding functions - sources of vortexes - are defined by the following expressions:

$$
\begin{aligned}
& f(\rho, \varphi, z)=f(\rho, z) e^{i m_{1} \varphi} \\
& \psi(\rho, \varphi)=\psi(\rho) e^{i m_{2} \varphi} .
\end{aligned}
$$

The radiated field, in accordance with expression (8), has the form

$$
\begin{aligned}
& U(\rho, \varphi, z)=\int_{0}^{+\infty} \int_{0}^{2 \pi} \psi\left(\rho^{\prime}\right) e^{i m_{1} \varphi} G\left(\rho, \rho^{\prime}, \varphi, \varphi^{\prime}, z\right) \rho^{\prime} d \rho^{\prime} d \varphi^{\prime}+ \\
& +\int_{0}^{z} \int_{0}^{+\infty} \int_{0}^{+\infty} f(\rho, z) e^{i m_{2} \varphi} G\left(\rho, \rho^{\prime}, \varphi, \varphi^{\prime}, z-z^{\prime}\right) \rho^{\prime} d \rho^{\prime} d \varphi^{\prime} d z^{\prime}
\end{aligned}
$$


where the cylindrical Green's function $G(\rho, \varphi, z)$ is defined as

$$
G\left(\rho, \rho^{\prime}, \varphi, \varphi^{\prime}, z\right)=-\frac{1}{4 \pi z} \exp \left\{\frac{i k}{2 z}\left[\rho^{2}+\left(\rho^{\prime}\right)^{2}-2 \rho \rho^{\prime} \cos \left(\varphi-\varphi^{\prime}\right)\right]\right\}
$$

In representation (12), it is easy to perform integration over angle $\varphi^{\prime}$ if we use the integral representation of the Bessel function

$$
J_{n}(x)=\frac{i^{-n}}{2 \pi} e^{i n \varphi} \int_{0}^{2 \pi} e^{-i x \cos (\theta-\varphi)+i n \theta} d \theta
$$

which is relevant for an arbitrary angle $\varphi$. As a result, the radiation field defined by expression (11) can be represented in the following form

$$
U(\rho, \varphi, z)=A\left(\rho, z, m_{1}\right) e^{i m_{1} \varphi}+B\left(\rho, z, m_{2}\right) e^{i m_{2} \varphi}
$$

where the functions $A\left(\rho, z, m_{1}\right)$ and $B\left(\rho, z, m_{2}\right)$ are given by the relations:

$$
\begin{aligned}
& A\left(\rho, z ; m_{1}\right)=\frac{(-i)^{m_{1}} \exp \left\{\frac{i k \rho^{2}}{2 z}\right\}}{2 z} \int_{0}^{+\infty} \psi\left(\rho^{\prime}\right) \exp \left\{\frac{i k \rho^{\prime}}{2 z}\right\} \times J_{m_{1}}\left(\frac{k \rho \rho^{\prime}}{z}\right) d \rho^{\prime} \\
& B\left(\rho, z ; m_{2}\right)=(-i)^{m_{2}} \int_{0}^{z} \int_{0}^{+\infty} f\left(\rho^{\prime}, z^{\prime}\right) \exp \left\{\frac{i k\left[\rho^{2}+\rho^{\prime 2}\right]}{2\left(z-z^{\prime}\right)}\right\} \times J_{m_{2}}\left(\frac{k \rho \rho^{\prime}}{z-z^{\prime}}\right) d \rho^{\prime} d z^{\prime}
\end{aligned}
$$

Expressions (14), (15), and (16) give a solution to the problem of propagation of radiation carrying an angular momentum upon excitation of a wave by surface and volumetric sources. Solution (14), due to the linearity of the original equations, is generalized to the case when the radiation sources are represented by vortex superpositions:

$$
\begin{aligned}
& f(\rho, \varphi, z)=\sum_{\xi=1} f_{\xi}(\rho, z) e^{i m_{\xi} \varphi} \\
& \psi(\rho, \varphi, z)=\sum_{k=1} \psi_{k}(\rho) e^{i m_{k} \varphi}
\end{aligned}
$$

In this case, instead of (14), we get

$$
U(\rho, \varphi, z)=\sum_{\xi} A_{\xi}\left(\rho, z, m_{\xi}\right) e^{i m_{\xi} \varphi}+\sum_{k} B_{k}\left(\rho, z, m_{k}\right) e^{i m_{k} \varphi}
$$

where $A_{\xi}$ are determined in accordance with expressions (15) and (16). Note that in expressions (17)-(18) $\xi$ and $k$ are just indexes of summation.

Thus, topological charges in the case of vortex emission by surface and volumetric sources are conserved during propagation in the linear regime.

\section{Three-wave mixing in a nonlinear medium}

The propagation of electromagnetic waves in nonlinear media is determined by the polarization vector $\vec{P}$, which in symbolic scalar form can be represented as an expansion in powers of the electric field strength.

$$
P=E_{0}\left(\chi^{(1)} E+\chi^{(2)} E^{2}+\chi^{(3)} E^{3}+\ldots\right)
$$

where $\chi^{(1)} \sim 1$ is the linear polarizability, $\chi^{(2)} \sim 10^{-12} \div 10^{-11}$ is the quadratic polarizability, and $\chi^{(3)} \sim 10^{-22} \div 10^{-21}$ is the cubic Kerr polarizability. 
Let us consider the process of three-wave mixing, in which the quadratic term plays a decisive role. In this case, describing an isotropic medium, the nonlinear part of the polarization vector can be represented as

$$
\vec{P}_{N L}=\varepsilon_{0} \chi^{(2)} E \cdot \vec{E}
$$

Hence, propagation of electromagnetic waves in a nonlinear medium will be described by the following equation:

$$
\nabla^{2} \vec{E}-\frac{\varepsilon}{c^{2}} \frac{\partial^{2} \vec{E}}{\partial t^{2}}=\frac{1}{c^{2} \varepsilon_{0}} \frac{\partial^{2} \vec{P}_{N L}}{\partial t^{2}}
$$

where $\vec{E}$ is the vector of the electric field strength, $c$ is the speed of light in vacuum, $\varepsilon$ is the dielectric constant of the medium, $\vec{P}_{N L}(x, y, z, t)$ is the vector of nonlinear polarization.

For narrowband signals with a carrier frequency $\omega$ used in telecommunication systems (i.e. when $\Delta \omega<<\omega$, where $\Delta \omega$ is the spectrum width), the commonly used approach to solve equation (21) is to use the method of SVEA, according to which the electric field is represented in the form

$$
E(x, y, z, t)=\tilde{E}(x, y, z) e^{i k z-i \omega t}
$$

where $k=\frac{\omega}{c} \sqrt{\varepsilon}, \tilde{E}(x, y, z)$ is the z-coordinate function slowly varying on the wavelength scale, satisfying the condition:

$$
\frac{\partial^{2} \tilde{E}}{\partial z^{2}}<<k \frac{\partial \tilde{E}}{\partial z}
$$

In the case when two waves with frequencies $\omega_{1}$ and $\omega_{2}$ propagate in a nonlinear medium with quadratic polarization, so that

$$
E=E_{1} e^{i\left(k_{1} z-\omega_{1} t\right)}+E_{1}^{*} e^{-i\left(k_{1} z-\omega_{1} t\right)}+E_{2} e^{i\left(k_{2} z-\omega_{2} t\right)}+E_{2}^{*} e^{-i\left(k_{2} z-\omega_{2} t\right)}
$$

In this case, the quadratic polarization is represented as

$$
P_{N L}^{(2)}=\varepsilon_{0} \chi^{(2)}\left[E_{1} E_{1}^{*}+E_{2} E_{2}^{*}+E_{1}^{2} e^{i\left(2 \omega_{1}-2 k_{1} z\right)}+\ldots E_{1} E_{2}^{*} e^{i\left(\omega_{1}-\omega_{2}\right) t-\left(k_{1}-k_{2}\right) z}\right]
$$

Polarization (25), due to the dipole radiation mechanism, leads to the following processes in the medium that occur at different frequencies: $\omega_{3}=0$ - optical rectification; $\omega_{3}=2 \omega_{1}$ or $\omega_{3}=2 \omega_{2}$ - frequency doubling; $\omega_{3}=\omega_{1}+\omega_{2}-$ generation of the sum frequency; $\omega_{3}=\omega_{1}-\omega_{2}-$ generation of the difference frequency. From the point of view of radio photonics, the process of the difference frequency generation leads to the generation of radio waves as a result of mixing the initial waves of the optical range. Generation of the difference frequency when searching for a solution to equation (21) in the form (22) leads to a system of equations:

$$
\begin{aligned}
& \nabla_{\perp}^{2} \tilde{E}_{1}+2 i k_{1} \frac{\partial \tilde{E}_{1}}{\partial z}=-\chi^{(2)} \frac{\omega_{1}^{2}}{c^{2}} \tilde{E}_{2} \tilde{E}_{3} e^{-i\left(k_{3}+k_{2}-k_{1}\right) z} \\
& \nabla_{\perp}^{2} \tilde{E}_{2}+2 i k_{2} \frac{\partial \tilde{E}_{2}}{\partial z}=-\chi^{(2)} \frac{\omega_{1}^{2}}{c^{2}} \tilde{E}_{2} \tilde{E}_{3}^{*} e^{-i\left(k_{3}+k_{2}-k_{1}\right) z} \\
& \nabla_{\perp}^{2} \tilde{E}_{3}+2 i k_{3} \frac{\partial \tilde{E}_{3}}{\partial z}=-\chi^{(2)} \frac{\omega_{3}^{2}}{c^{2}} \tilde{E}_{1} \tilde{E}_{2}^{*} e^{-i\left(k_{3}+k_{2}-k_{1}\right) z}
\end{aligned}
$$


where $\tilde{E}_{1}, \tilde{E}_{2}, \tilde{E}_{3}$ are the envelopes corresponding to signals with frequencies $\omega_{1}, \omega_{2}, \omega_{3}$ and wave numbers $k_{1}, k_{2}, k_{3}, \nabla_{\perp}^{2}=\frac{\partial^{2}}{\partial x^{2}}+\frac{\partial^{2}}{\partial y^{2}}$ is the transverse component of the Laplace operator.

Let us estimate the influence of nonlinear effects on changes in electromagnetic fields during propagation along a nonlinear medium of length $\Delta z$ in the case when the process is initiated by laser radiation of power $P_{0}$ with beams of radius $\sigma_{0}$.

$$
\left.\left.\tilde{E}_{1}\right|_{z=0} \approx \tilde{E}_{2}\right|_{z=0}=E_{0}
$$

where $E_{0}=\sqrt{\frac{P_{0} W_{0}}{\pi \sigma_{0}^{2}}}, W_{0}=\sqrt{\frac{\mu_{0}}{\varepsilon_{0}}}$ is the wave impedance of vacuum, $\mu_{0}$ is the universal magnetic constant, $\varepsilon_{0}$ is the universal electric constant.

Now we introduce the following dimensionless quantities:

$$
\bar{z}_{i}=k_{i} z, \bar{x}=\frac{x}{\sigma_{0}}, \bar{y}=\frac{y}{\sigma_{0}}, \bar{E}_{i}=\frac{\tilde{E}_{i}}{E_{0}}
$$

For an approximate physical estimate of the change in electric fields from system (26), we obtain

$$
\frac{\Delta \bar{E}_{1}}{\Delta \overline{z_{1}}} \sim \gamma \bar{E}_{1} \bar{E}_{3}, \frac{\Delta \bar{E}_{2}}{\Delta \overline{z_{2}}} \sim \gamma \bar{E}_{2} \bar{E}_{3}, \frac{\Delta \bar{E}_{3}}{\Delta \bar{z}_{3}} \sim \gamma \bar{E}_{1} \bar{E}_{2}
$$

In equations (29), $\gamma$ is a dimensionless parameter characterizing nonlinearity:

$$
\gamma=\sqrt{\frac{W_{0} P_{0}}{\pi \sigma_{0}^{2}}} \chi^{(2)}
$$

Taking into account that $\Delta \bar{E}_{3}=\bar{E}_{3}$, the system of equations (29) yields estimates for the relative change in optical fields $\xi=1,2$ :

$$
\frac{\Delta E_{\xi}}{E_{\xi}} \leq \gamma^{2} \frac{(\Delta z)^{2}}{\lambda_{\xi} \cdot \lambda_{3}}
$$

In the conditions typical for telecommunication applications, the initial physical quantities have the following values: $P_{0} \sim 1$ Watt, $\sigma_{0} \sim 1 \mathrm{~mm}, \Delta z \sim 1-10 \mathrm{~cm}, \lambda_{1,2} \sim 1.5 \mu \mathrm{m}$.

The nonlinearity parameter is a very small value: $\gamma \sim 10^{-8}$, and the changes in optical fields in the entire terahertz range of $0.1-10 \mathrm{THz}$ are, in accordance with estimation (32), the following values (in order of magnitude):

$$
\begin{aligned}
& \frac{\Delta E_{1,2}}{E_{1,2}} \leq 10^{-11} \div 10^{-8}, \text { when } \Delta z \sim 1 \mathrm{~cm} \\
& \frac{\Delta E_{1,2}}{E_{1,2}} \leq 10^{-11} \div 10^{-6} \text {, when } \Delta z \sim 10 \mathrm{~cm}
\end{aligned}
$$

In this case, the change in the optical fields can be neglected, and the system of nonlinear equations (26), which describes the process of generation of the difference frequency, is reduced to a linear equation of the type (7):

$$
\nabla_{\perp}^{2} \tilde{E}_{3}+2 i k_{3} \frac{\partial \tilde{E}_{3}}{\partial z}=f(x, y, z)
$$

with the volumetric radiation source 


$$
f(x, y, z)=-\chi^{2} \frac{\omega_{3}^{2}}{c^{2}} \tilde{E}_{1} \tilde{E}_{2}^{*}
$$

if we reckon that the phase matching condition $k_{3}=k_{1}-k_{2}$ is satisfied. In expression (34), the fields $\tilde{E}_{1}$ and $\tilde{E}_{2}$ correspond to fields propagating in a linear medium.

The solution of equation (34) can be found according to expression (8) in which there is no surface source:

$$
\tilde{E}_{3}=\int_{0}^{z} \int_{-\infty}^{+\infty} \int_{-\infty}^{+\infty} G\left(x-x^{\prime}, y-y^{\prime}, z-z^{\prime}\right) f\left(x^{\prime}, y^{\prime}, z^{\prime}\right) d x^{\prime} d y^{\prime} d z^{\prime}
$$

and where the Green's function is determined by expression (9) in which it should be assumed $k=k_{3}$.

When the three-wave mixing process is initiated by laser radiation, the optical fields are represented by a set of Laguerre-Gauss (LG) beams:

$$
\tilde{E}_{\xi}=\Phi_{L G}\left(p, z, m_{\xi}, n\right) \cdot e^{i m_{\xi} \varphi}
$$

The complex amplitude of laser beams can have different shapes [42-45], in particular, the set of Laguerre -Gauss beams can be described by the following distribution:

$$
\begin{aligned}
& \Phi_{\mathrm{LG}}(\varrho, z, m, n)=\frac{\sigma_{0}}{\sigma(z)} \exp \left[-i \frac{k \varrho^{2}}{2 R(z)}-\frac{\varrho^{2}}{\sigma^{2}(z)}\right] \times\left(\frac{\sqrt{2} \varrho}{\sigma(z)}\right)^{|m|} L_{n}^{|m|}\left(\frac{2 \varrho^{2}}{\sigma^{2}(z)}\right) \times \\
& \times \exp \left[i(2 n+m+1) \operatorname{arctg} \frac{z}{z_{R}}\right],
\end{aligned}
$$

where $R(z)=z\left(1+\frac{z_{R}^{2}}{z^{2}}\right)$ is the radius of curvature of the parabolic front of the beam, $\sigma(z)=\sigma_{0} \sqrt{\left(1+\frac{z_{R}^{2}}{z^{2}}\right)}$ is the radius of the beam, $\sigma_{0}$ is the radius of the beam waist, $z_{R}=\frac{\pi \sigma_{0}^{2}}{\lambda}$ is the Rayleigh confocal parameter, and $L_{n}^{|m|}$ is the associated Laguerre polynomial.

Since the influence of the radial order of the set of Gauss-Laguerre beams $n$ is not considered in this work, the class of LG field distributions can be narrowed down by setting $n=0$.

Passing in the integral representation (35) to the limit $z \rightarrow 0$ and taking into account that the relation holds

$$
\lim _{z \rightarrow z^{\prime}} \frac{k}{2 \pi i\left(z-z^{\prime}\right)} \exp \left\{\frac{i k\left[\left(x-x^{\prime}\right)^{2}+\left(y-y^{\prime}\right)^{2}\right]}{2\left(z-z^{\prime}\right)}\right\}=\delta\left(x-x^{\prime}\right) \cdot \delta\left(y-y^{\prime}\right)
$$

where $\delta(x)$ and $\delta(y)$ are the Dirac delta functions, we obtain an approximate solution

$$
E_{3}=\frac{\sqrt{P_{1} P_{2} W_{0}}}{\pi \sigma_{0}^{2}} n !\left(\sqrt{\frac{2 \rho}{\sigma_{0}}}\right)^{\left|m_{1}\right|+\left|m_{2}\right|} L_{n}^{m_{1}}\left(\frac{2 \rho^{2}}{\sigma_{0}^{2}}\right) L_{n}^{m_{2}}\left(\frac{2 \rho^{2}}{\sigma_{0}^{2}}\right) e^{-\frac{\rho^{2}}{\sigma_{0}^{2}}} e^{i\left(m_{1}-m_{2}\right) \varphi} z
$$

where $P_{1}$ and $P_{2}$ are the power of optical radiation at frequencies $\omega_{1}$ and $\omega_{2}$.

To derive the expression (39), the first nonzero term was taken into account in the expansion of field (34) in powers of the coordinate $z$ in a Taylor series. 
In the case of generating a vortex with a topological charge $m_{3}=1$, when $m_{1}=1, m_{2}=0$ for the power of the radio signal, from (38) one can obtain the ratio:

$$
P_{3}=\frac{W_{0}}{4 \pi^{3 / 2}}\left[\chi^{(2)}\right]^{2} \frac{k_{3}^{2}(\Delta z)^{2}}{\sigma_{0}^{2}} P_{1} P_{2}
$$

\section{Modeling of nonlinear processes by a modified method of slowly varying envelope approximation}

In [46], a modified method of slowly varying envelope approximation was developed based on the factorization of a slowly varying amplitude into two factors, one of which is forced to obey the parabolic equation, and the second one depends only on the coordinate in the propagation direction and obeys a differential equation, which makes it possible to improve accuracy when describing the three-wave mixing process. In this method, in contrast to the search for a solution to equation (21) in the form (22), the envelope $\tilde{E}(x, y, z)$ is represented in a more detailed multiplicative form so that

$$
\vec{E}(x, y, z, t)=\vec{A}(z) \psi(x, y, z) e^{i k z-i \omega t}
$$

where $\psi(x, y, z)$ is a solution to the parabolic equation:

$$
\nabla_{\perp}^{2}+2 i k \frac{\partial \psi}{\partial z}=0
$$

If the electric field represents a certain superposition of waves with frequencies $\omega_{v}$, wavenumbers $k_{\mathrm{v}}$ and directions of polarization $\vec{e}_{\mathrm{v}}$, then the electric field strength and polarization can be represented in the form

$$
\begin{gathered}
\vec{E}=\sum_{\mathrm{v}} \vec{e}_{\mathrm{v}} A_{\mathrm{v}}(z) \Psi_{\mathrm{v}}(x, y, z) e^{i k_{\mathrm{v}} z-i \omega_{\mathrm{v}} t}, \\
\vec{P}_{N L}=\sum_{\mathrm{v}} \vec{e}_{\mathrm{v}} P_{\mathrm{v}}(z) e^{i i_{\mathrm{v}} z-i \omega_{\mathrm{v}} t} .
\end{gathered}
$$

Then from equation (21) it follows:

$$
\frac{\partial A_{v}}{\partial z}\left[\frac{\partial \Psi_{v}}{\partial z}+i k_{v} \Psi_{v}\right] e^{i k_{v} z}=\frac{\omega_{v}^{2}}{2 c^{2} \varepsilon_{0}} P_{v} .
$$

As a result of the scalar representation, the electric field in the medium can be written as a sum of three waves describing the propagation of vortex beams with projections of angular momentum $m_{1}, m_{2}$, and $m_{3}$ at frequencies $\omega_{1}, \omega_{2}$, and $\omega_{3}$ :

$$
\begin{aligned}
& E=A_{1}(z) \Phi_{1}(x, y, z) e^{i k_{1} z-i \omega_{1} t+i m_{1} \varphi}+A_{2}(z) \Phi_{2}(x, y, z) e^{i k_{2} z-i \omega_{2} t+i m_{2} \varphi}+ \\
& +A_{3}(z) \Phi_{3}(x, y, z) e^{i k_{3} z-i \omega_{3} t+i m_{3} \varphi}+\text { c.c., }
\end{aligned}
$$

where $k_{1}=\frac{\omega_{1}}{c} \sqrt{\varepsilon_{1}}, k_{2}=\frac{\omega_{2}}{c} \sqrt{\varepsilon_{2}}, k_{3}=\frac{\omega_{3}}{c} \sqrt{\varepsilon_{3}} ; \quad \varepsilon_{1}, \varepsilon_{2}$ and $\varepsilon_{3}$ are the values of dielectric constants at frequencies $\omega_{1}, \omega_{2}$, and $\omega_{3}$, respectively; $\Phi_{\xi}$ are the distributions of the set (36) corresponding to the azimuthal indices $m \xi$.

Now we can write scalar nonlinear polarization in the form:

$$
P_{N L}=\sum_{v=1}^{3} P_{v} e^{i k_{v} z-i \omega_{v} t+i m_{v} \varphi}+\text { c.c., }
$$


which corresponds to equation (44) in the scalar representation for vortex fields. For a nonlinear medium, represented in the form (20), we get:

$$
\begin{aligned}
& P_{N L}=d\left[A_{2} A_{3} \Phi_{2} \Phi_{3} e^{-i \omega_{1} t+i\left(k_{3}+k_{2}\right) z+i\left(m_{2}+m_{3}\right) \varphi}+\right. \\
& +A_{1} A_{3}^{*} \Phi_{1} \Phi_{3}^{*} e^{-i \omega_{2} t+i\left(k_{1}-k_{3}\right) z+i\left(m_{1}-m_{3}\right) \varphi}+ \\
& \left.+A_{1} A_{2}^{*} \Phi_{1} \Phi_{2}^{*} e^{-i \omega_{3} t+i\left(k_{1}-k_{2}\right) z+i\left(m_{1}-m_{2}\right) \varphi}+\text { c.c. }\right] .
\end{aligned}
$$

In expression (48), when squaring, we consider only the terms corresponding to the process of generating the difference frequency. Thus, in expression (48), one should consider:

$$
\omega_{1}=\omega_{3}+\omega_{2}, \omega_{2}=\omega_{1}-\omega_{3}, \omega_{3}=\omega_{1}-\omega_{2}
$$

Comparing expressions (47) and (48), we find:

$$
\begin{aligned}
& P_{1}=d A_{2} A_{3} \Phi_{2} \Phi_{3} e^{-i \omega_{1} t+i\left(k_{3}+k_{2}\right) z+i\left(m_{2}+m_{3}\right) \varphi} \\
& P_{2}=d A_{1} A_{3}^{*} \Phi_{1} \Phi_{3}^{*} e^{-i \omega_{2} t+i\left(k_{1}-k_{3}\right) z+i\left(m_{1}-m_{3}\right) \varphi} \\
& P_{3}=d A_{1} A_{2}^{*} \Phi_{1} \Phi_{2}^{*} e^{-i \omega_{3} t+i\left(k_{1}-k_{2}\right) z+i\left(m_{1}-m_{2}\right) \varphi}
\end{aligned}
$$

Using equation (45) for each of the propagating waves, we can get the system of equations:

$$
\begin{aligned}
& \frac{d A_{1}}{d z}\left[\frac{d \Phi_{1}}{d z}+i k_{1} \Phi_{1}\right]=\chi^{2} \frac{\omega_{1}^{2}}{c^{2}} A_{2} A_{3} \Phi_{2} \Phi_{3} e^{i \Delta k z} e^{i \Delta m \varphi} ; \\
& \frac{d A_{2}}{d z}\left[\frac{d \Phi_{2}}{d z}+i k_{2} \Phi_{2}\right]=\chi^{2} \frac{\omega_{2}^{2}}{c^{2}} A_{1} A_{3}^{*} \Phi_{1} \Phi_{3}^{*} e^{-i \Delta k z} e^{-i \Delta m \varphi} ; \\
& \frac{d A_{3}}{d z}\left[\frac{d \Phi_{3}}{d z}+i k_{3} \Phi_{3}\right]=\chi^{2} \frac{\omega_{3}^{2}}{c^{2}} A_{1} A_{2}^{*} \Phi_{1} \Phi_{2}^{*} e^{-i \Delta k z} e^{-i \Delta m \varphi},
\end{aligned}
$$

where $\Delta m=m_{1}-m_{2}-m_{3}, \Delta k=k_{3}+k_{2}-k_{1}$.

Analyzing the system of equations (51), we see that the left-hand sides of each equation do not depend on the angle $\varphi$, thus, solutions to system (51) exist when the condition $\Delta m=0$ is satisfied. Consequently, in a quadratic nonlinear medium, from the initial vortex beams carrying angular moments with azimuthal indices $m_{1}$ and $m_{2}$ at frequencies $\omega_{1}$ and $\omega_{2}$, a vortex beam of difference frequency $\omega_{3}=\omega_{1}-\omega_{2}$ is generated, which transfers angular momentum with a projection on the direction of propagation equal to the difference of projections $m_{3}=m_{1}-m_{2}$.

Following the method outlined in [5], we multiply each of the $v$-th equation of system (50) by a complex conjugate factor $\frac{d \Phi_{v}^{*}}{d z}-i k_{v} \Phi_{v}^{*}$ and integrate the equations over the cross section of the corresponding beams. Finally, to determine the amplitudes $A_{v}$, we obtain the system:

$$
\begin{aligned}
& \frac{d A_{1}}{d z}=-\chi^{2} \frac{\omega_{1}^{2}}{2 c^{2}} A_{2} A_{3} e^{i \Delta k z} I_{1} ; \\
& \frac{d A_{2}}{d z}=-\chi^{2} \frac{\omega_{2}^{2}}{2 c^{2}} A_{1} A_{3}^{*} e^{-i \Delta k z} I_{2} ; \\
& \frac{d A_{3}}{d z}=-\chi^{2} \frac{\omega_{3}^{2}}{2 c^{2}} A_{1} A_{2}^{*} e^{-i \Delta k z} I_{3},
\end{aligned}
$$

where the following notation is introduced: 


$$
\begin{aligned}
& I_{1}=\frac{\int_{0}^{\infty} \Phi_{2}\left(\varrho, z, m_{2}\right) \Phi_{3}\left(\varrho, z, m_{3}\right) B_{1}\left(\varrho, z, m_{1}\right) 2 \pi \varrho d \varrho}{F_{1}\left(z, m_{1}\right)} ; \\
& I_{2}=\frac{\int_{0}^{\infty} \Phi_{1}\left(\varrho, z, m_{1}\right) \Phi_{3}^{*}\left(\varrho, z, m_{3}\right) B_{2}\left(\varrho, z, m_{2}\right) 2 \pi \varrho d \varrho}{F_{2}\left(z, m_{2}\right)} ; \\
& I_{3}=\frac{\int_{0}^{\infty} \Phi_{1}\left(\varrho, z, m_{1}\right) \Phi_{2}^{*}\left(\varrho, z, m_{2}\right) B_{3}\left(\varrho, z, m_{3}\right) 2 \pi \varrho d \varrho}{F_{3}\left(z, m_{3}\right)} .
\end{aligned}
$$

In expressions (53), the functions $F_{v}\left(z, m_{v}\right)$ and $B_{v}\left(Q, z, m_{v}\right)$ are determined by the relations:

$$
\begin{gathered}
B_{v}=\frac{d \Phi_{v}^{*}}{d z}-i k_{v} \Phi_{v}^{*}, \\
F_{v}\left(z, m_{v}\right)=\int_{0}^{\infty}\left(\frac{d \Phi_{v}}{d z} \cdot \frac{d \Phi_{v}^{*}}{d z}+i k_{v}\left(\Phi_{v} \frac{d \Phi_{v}^{*}}{d z}-\Phi_{v}^{*} \frac{d \Phi_{v}^{*}}{d z}\right)+k_{v}^{2} \Phi_{v}^{*} \Phi_{v}\right) \pi \rho d \rho .
\end{gathered}
$$

The analysis of the influence of nonlinear effects given in Section 2 allows us to assert that under conditions typical for telecommunication applications, the system of nonlinear equations (52) is reduced to the third equation, in which $A_{1}=\sqrt{\frac{P_{1} W_{0}}{\pi \sigma_{0}^{2}}} ; A_{2}=\sqrt{\frac{P_{2} W_{0}}{\pi \sigma_{0}^{2}}}$.

As a result, the solution is determined by the integral

$$
A_{3}(z)=\chi^{(2)} \frac{\omega_{3}^{2}}{2 c^{2}} \frac{\sqrt{P_{1} P_{2}}}{\pi \sigma_{0}^{2}} \int_{0}^{z} I_{3}^{(z)} d z
$$

\section{Simulation of the propagation of optical vortexes}

Simulation of the propagation processes of vortexes in the optical range and their transformation into vortexes in the terahertz radio range was carried out using the numerical solution of the system of equations (52) and on the basis of the integral representation (56). The Runge-Kutta method in the Matlab package of mathematical modeling is used to solve system (52). It should be noted that the simulation results based on the system of equations (52) and integral representation (55) under the conditions defined in Section 2 are practically identical, the order of differences is $\approx 10^{-12}$.

The difference frequency generation process was investigated in the radio frequency range $100 \mathrm{GHz}-700 \mathrm{GHz}$. The optical radiation corresponded to the C-band wavelengths. The pump laser power was $P_{1}=P_{2}=1 \mathrm{~W}$. The effective nonlinear susceptibility of the nonlinear medium in which the conversion process takes place is $\chi^{(2)}=1.5 \times 10^{-12}$, the refractive indices at optical frequencies are $n_{1}=n_{2}=2.2$, at radio frequencies $n_{3}=2.5$.

To generate radio vortex beams with a topological charge $m_{3}=1$, Gauss-Laguerre laser modes with radial indices $n_{1}=n_{2}=0$ and azimuthal indices $m_{1}=1, m_{2}=0$ were used. The simulated system is shown in Fig. 1. 


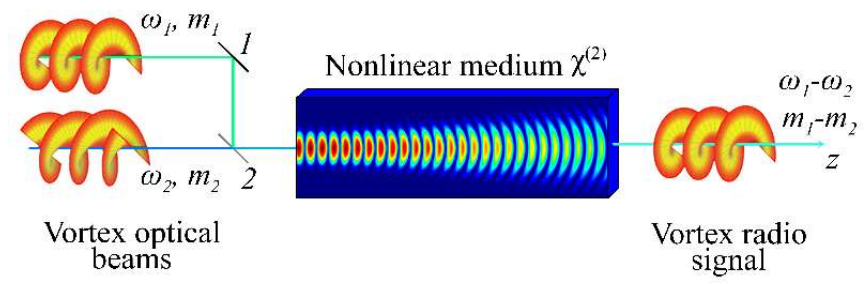

Figure 1. Simulated scheme for converting vortex beams: 1 - opaque mirror, 2 - semitransparent mirror.

From the point of view of technical applications of the results, it should be interesting to study the nature of the change in the modulus of the amplitude of the obtained radio-vortex beam, obeying relation (21), with a change in the length of the nonlinear crystal, which is shown in Fig. 2. Calculations were carried out for a radio vortex beam with a topological charge $m_{3}=1$ depending on the length of the nonlinear medium for different values of the waist radii of the pump laser beams. It follows from the data obtained that at small lengths of the nonlinear medium, a linear dependence of the amplitude on the length is observed, which is consistent with expression (32). With an increase in the length of the nonlinear crystal, the amplitudes of the vortex radio beams reach the corresponding asymptotic values.

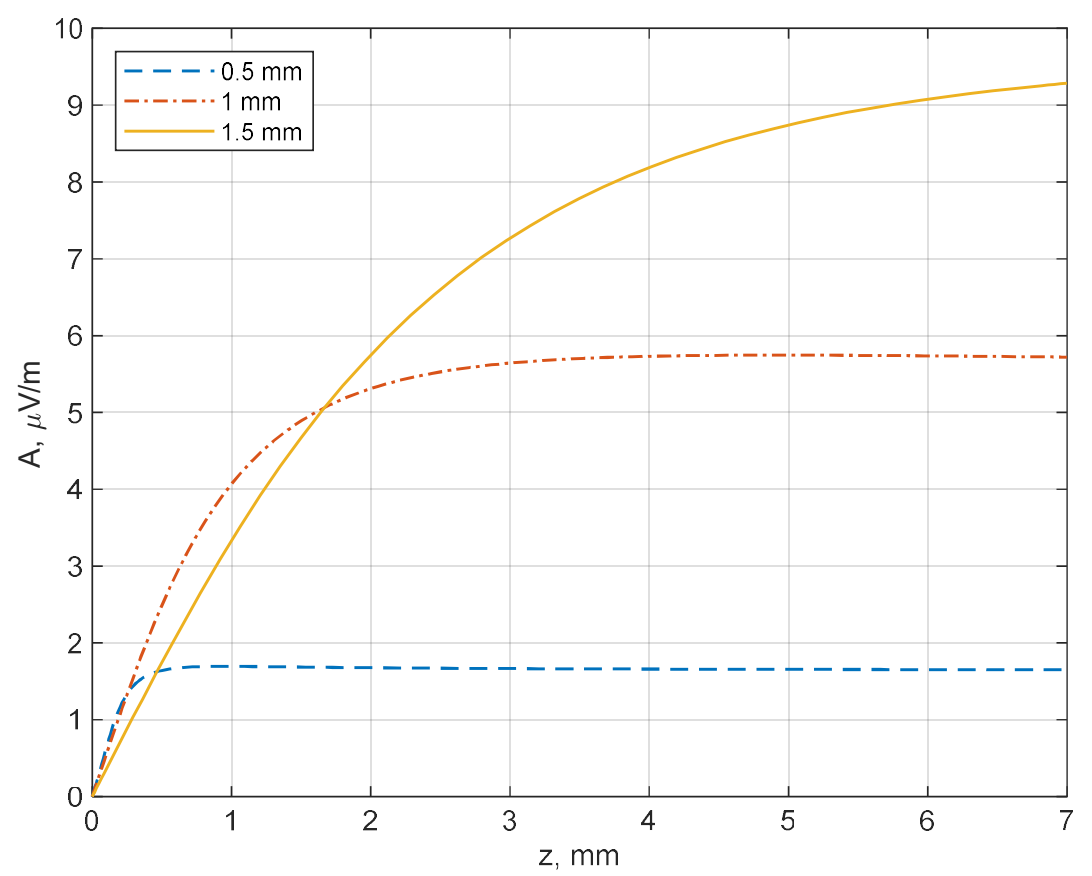

Figure 2. Dependence of the modulus of a vortex radio beam $A$ on the length of a nonlinear me$\operatorname{dium} z$ for three values of the waist radius of laser beams.

Fig. 3 shows the dependence of the efficiency index $\eta$ of the vortex radio beam generation on the length of the crystal. The efficiency index, in accordance with the work [6], is determined by the ratio having the unit of $1 / W$ :

$$
\eta=\frac{P_{3}}{P_{1} \cdot P_{2}}
$$

where $P_{1}$ and $P_{2}$ are the powers of the optical pumping lasers, $P_{3}$ is the power of the vortex radio beam. The choice of this ratio to efficiency estimation is due to the fact that these 
values are independent of the power of the original optical beams. Expression (40) confirms this fact, as well as the quadratic dependence on the length of the nonlinear medium in the region of small values.

Calculations show that the dependences of the amplitude $A_{3}$ of the difference frequency and the efficiency index $\eta$ on the crystal length for different values of the waist radius have a similar asymptotic character.

Fig. 4 shows the dependence of the efficiency index of generation of a radio vortex beam on the frequency difference between the pump lasers. With an increase in the frequency difference, the value of $\eta$ noticeably increases, which is due to the dipole radiation mechanism, as noted above.

Fig. 5 shows the dependence of the efficiency index on the waist radius of the laser beams. This dependence has an extreme nature, i.e. for a given pump power, there is a value of the beam waist radius at which the maximum radiation conversion efficiency is observed. This dependence is caused by the extreme dependence of the intensity of Gaussian beams on the waist radius, which at a given radiation power $P$ can be represented as:

$$
I \sim \frac{P}{\sigma^{2}} e^{-\frac{\rho^{2}}{\sigma^{2}}} .
$$

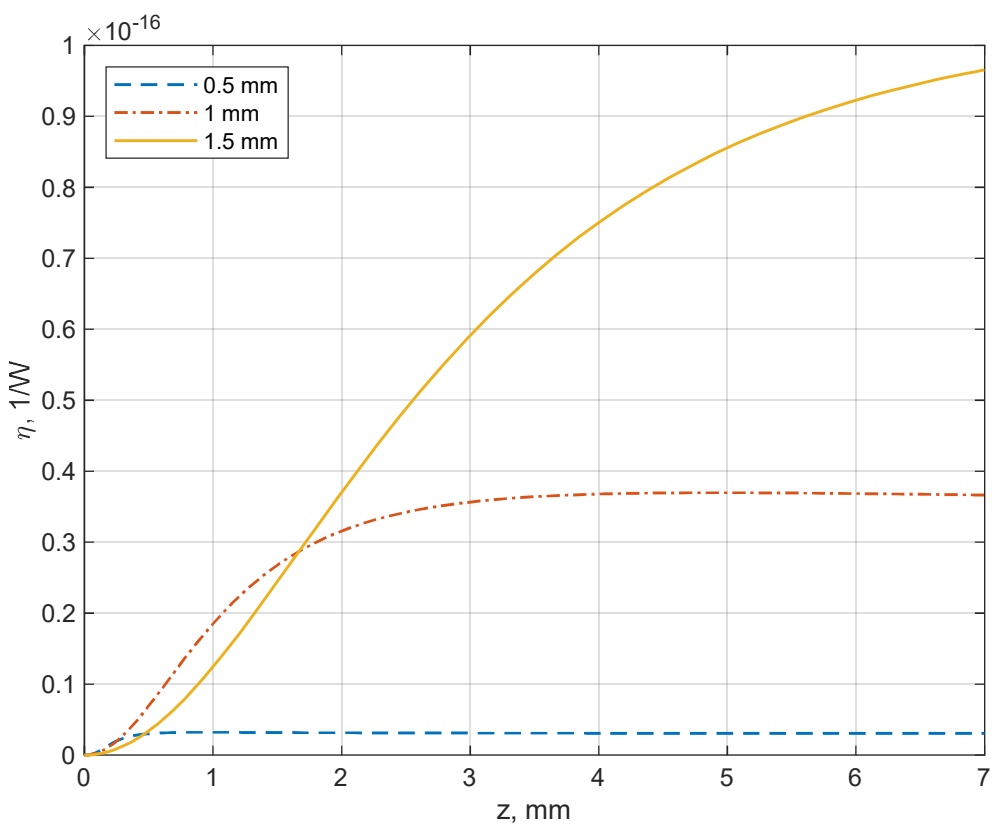

Figure 3. Dependence of the efficiency exponent $\eta$ of a radio vortex beam on the length of a nonlinear medium $z$ for three values of the waist radius of laser beams.

Thus, it is shown that with an increase in the length of the nonlinear medium, an asymptotic behavior of the efficiency index of the considered conversion is observed, which makes it possible to determine the geometric dimensions of the converting device. With an increase in the frequency difference, the generation efficiency increases. The performed calculations allow one to select the technical parameters of the converter depending on the system under consideration. The observed dependences are in qualitative agreement with the work [6]. 


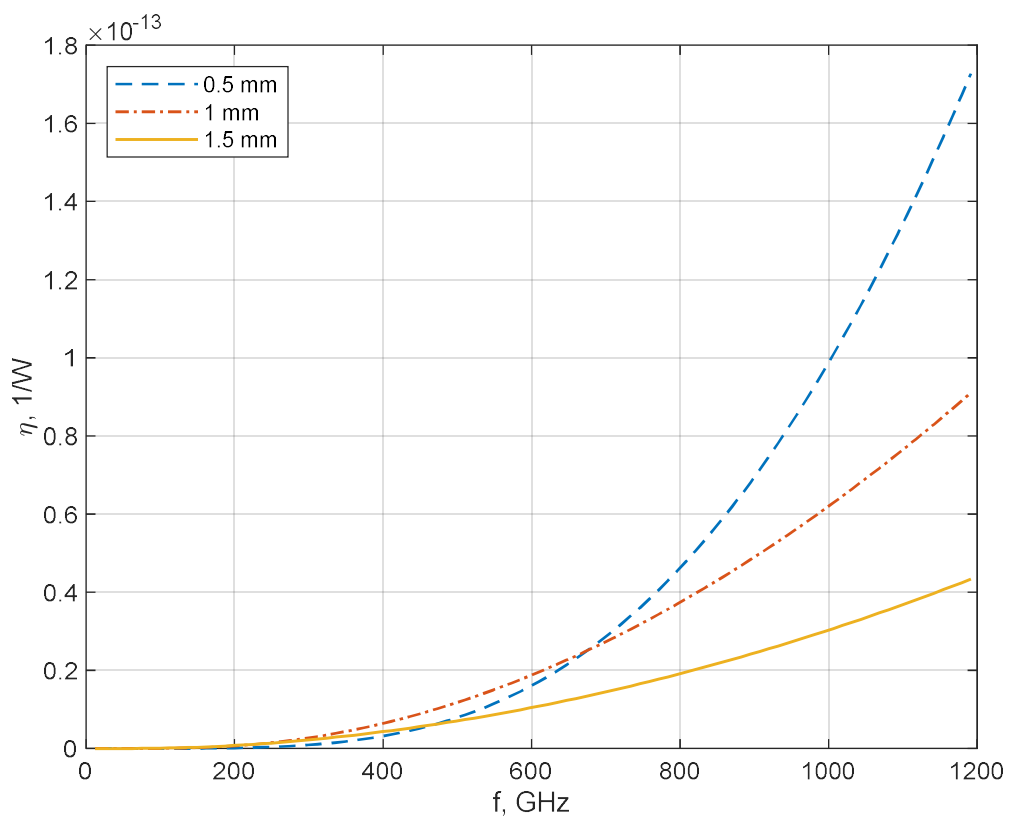

Figure 4. Dependence of the efficiency exponent $\eta$ of a radio vortex beam on the difference frequency $f$ of laser beams for three different values of the laser beam waist radius.

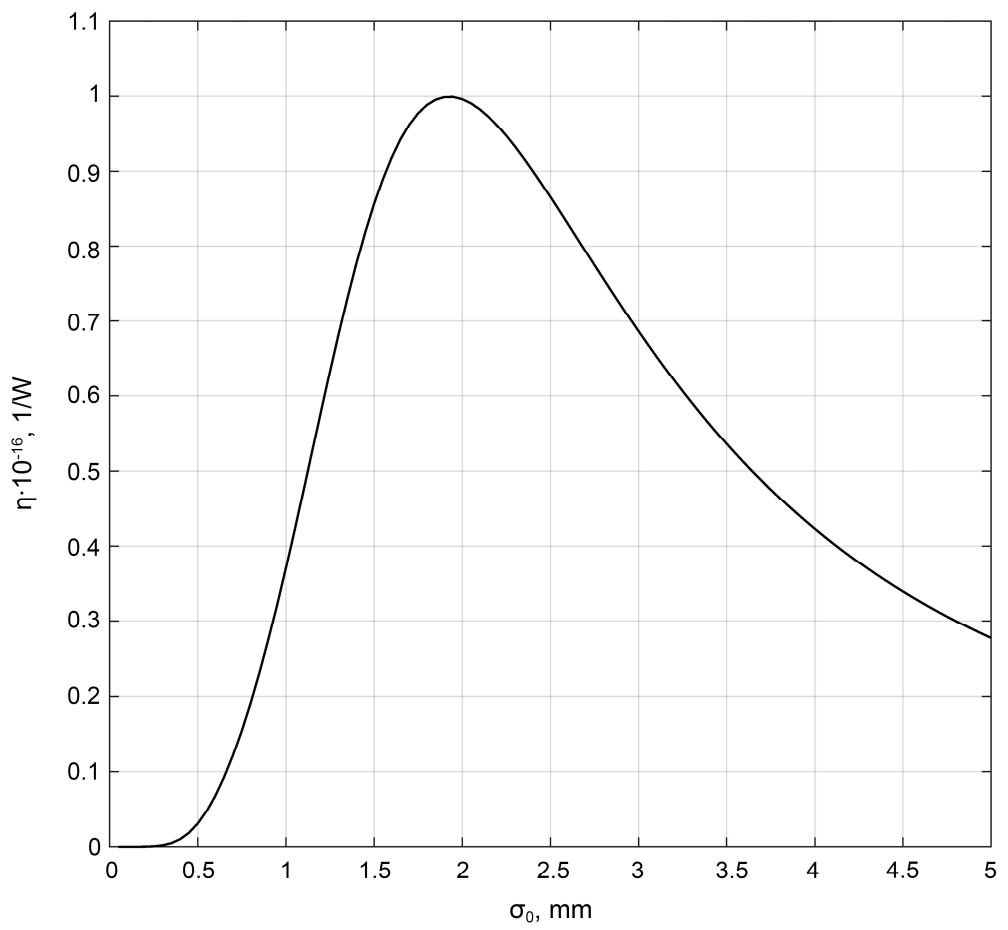

Figure 5. Dependence of the efficiency index of generation $\eta$ of a radio vortex beam on the waist radius $\sigma_{0}$ of laser beams.

The results obtained in this research can be used to construct promising high-speed hybrid RoF transmission systems [19], mainly in the submillimeter and microwave ranges, using the orbital angular momentum as an additional parameter for encoding and multiplexing multimedia data. The DFG process described in this paper will make it possible to avoid complex systems for converting vortex optical beams into radio beams while maintaining the vortex wavefront of an electromagnetic wave. 


\section{Conclusions}

In this paper, the propagation and conversion of vortexes in the optical range in linear and nonlinear media based on a modified model of three-wave mixing of optical radiation in a nonlinear medium with nonlinear second-order permeability are considered, the process of converting vortex beams of the optical range into vortex beams of the radio range is also investigated. It is shown that when the difference frequency is generated, the topological charge of the generated vortex beam is equal to the difference between the topological charges of the vortex beams of optical pump waves. The results of the process simulation show the asymptotic behavior of the lasing efficiency index as a function of the length of the nonlinear medium, an increase in the lasing efficiency with an increase in the frequency difference that determines the frequency of the radio vortex beam, and also the extreme behavior of the efficiency exponent on the waist radius of the pump laser beams. It should be noted that the use of Bessel-Gauss beams with fractional topological charge may turn out to be a promising direction of work in this area [9, 18].

Author Contributions: Conceptualization, formal analysis and methodology: V.H.B., R.R.N.; funding acquisition, I.K.M., A.Zh.S.; software and visualization, A.R.G.; writing-original draft preparation, review and editing, V.H.B.; project administration, A.Kh.S. All authors have read and agreed to the published version of the manuscript.

Funding: This work was partially supported by the Ministry of Science and Higher Education of the Russian Federation for research under the State Assignment of FSBEI HE USATU No. FEUE-2020-0007 on the topic "Theoretical foundations of modeling and semantic analysis of the processes of transformation of vortex electromagnetic fields in infocommunication systems" in the parts "Three-wave mixing in a nonlinear medium", "Modeling of nonlinear processes by a modified method of slowly varying envelope approximation" and "Simulation of the propagation of optical vortexes" and partially supported the Grant of the Russian Science Foundation (Project No. 19-49-04112) in the part "Transformation of vortex compositions in linear media"; A.Zh.S. was funded by the Ministry of Science and Higher Education of the Russian Federation (Agreement No. 075-03-2020-051, Topic No. fzsu-2020-0020, FOKRAT) .

Conflicts of Interest: The authors declare no conflict of interest.

\section{References}

1. Ng'oma, A. Radio-over-fiber techniques for millimeter wave wireless applications. IEEE International Topical Meeting on Microwave Photonics. 2015, pp. 1-4 [CrossRef]

2. Ruan, Z.; Veronis, G.; Vodopyanov, K. L.; Fejer, M. M.; Fan, S. Enhancement of optics-to-THz conversion efficiency by metallic slot waveguides. SPIE Photonics West 2010, January 23-28 2010, San Francisco, CA, Proceedings of the SPIE, 7582, 75820W, 2010. [CrossRef]

3. Petrov, E.V.; Manyizov, B.I.; Bushuev, V.A. Generation of terahertz difference frequency signals in a system of two-periodic one-dimensional photonic crystals in Quantum electronics, Russia, 2007, 37(4), 358-362. [CrossRef]

4. Murata, H.; Okamura, Y. Detection of an Optical Signal Using Difference Frequency Generation in a Periodically Poled LiTaO3 Microwave Waveguide Advances in OptoElectronics. 2008, 587091, 1-6. [CrossRef]

5. Qasymeh M. Terahertz Generation in Nonlinear Plasmonic Waveguides. IEEE Journal of Quantum Electronics 2016, 52(4), 1-7. [CrossRef]

6. De Regis, M.; Consolino, L.; Bartalini, S.; De Natale, P. Waveguided Approach for Difference Frequency Generation of Broadly-Tunable Continuous-Wave Terahertz Radiatio. Applied Sciences, 2018, 8(12), 2374. [CrossRef]

7. Courtial, J.; Dholakia, K.; Allen, L.; Padgett, M. Second-harmonic generation and the conservation of orbital angular momentum with high-order Laguerre-Gaussian modes. Physical Review A 1997, 56(5), 4193 [CrossRef]

8. Shao, Guang-hao; Wu, Zi-jian; Chen, Jin-hui; Xu, Fei; Lu, Yan-qing. Nonlinear frequency conversion of fields with orbital angular momentum using quasi-phase-matching. Physical Review A 2013, 88, 063827. [CrossRef]

9. Ni, R; Niu, YF; Du, L; Hu, XP; Zhang, Y; Zhu, SN. Topological Charge Transfer In Frequency Doubling Of Fractional Orbital Angular Momentum State. Applied Physics Letters 2016; 109, 151103. [CrossRef]

10. Zhou, Z-Y; Ding, D-Sh; Jiang, Y-K; Li, Y; Shi, Sh; Wang, X-Sh; Shi, B-S; Guo, G-C. Orbital Angular Momentum Light Frequency Conversion And Interference With Quasi-Phase Matching Crystals. Optics Express 2014, 22(17), 10.1364/OE.22.020298. [CrossRef]

11. Li, Y; Zhou, Z-Y; Ding, D-Sh; Shi, B-S; Sum Frequency Generation With Two Orbital Angular Momentum Carrying Laser Beams. Journal of the Optical Society of America 2015, 32(3), 407-411. [CrossRef] 
12. Fang, X; Wei, D; Yang, G; Wei, D; Ni, R; Zhang, Y; Hu, HP; Zhu, SN; Xiao, M. Nonlinear optical conversion of the orbital angular momentum of light in a PPLT crystal. Asia Communications and Photonics Conference 2016, AS1J.4 [CrossRef]

13. Fang, X; Wei, D.; Wang, Y. M. ; Wang, H. J.; Zhang, Y.; Hu, X. P.; Zhu, S. N.; Xiao, M. Conical third-harmonic generation in a hexagonally poled LiTaO3 crystal. Appl. Phys. Lett. 2017, 110, 111105.

14. Lin, Y.-C.; Nabekawa, Y.; Midorikawa, K. Conical third-harmonic generation of optical vortex through ultrashort laser filamentation in air, Opt. Express 2016, 24, 14857-14870.

15. Fang, X.; Yang, G.; Wei, D.; Wei, D.; Ni, R.; Ji, W.; Zhang, Y.; Hu, X.; Hu, W.; Lu, Y. Q.; Zhu, S. N.; Xiao, M. Coupled orbital angular momentum conversions in a quasi-periodically poled LiTaO3 crystal. Opt. Lett. 2016, 41(6), 1169-1172.

16. Wei, D.; Guo, J. L.; Fang, X. Y.; Wei, D. Z.; Ni, R.; Chen, P.; Hu, X. P. ; Zhang, Y.; Hu, W.; Lu, Y. Q.; Zhu, S. N.; Xiao, M. Multiple generations of high-order orbital angular momentum modes through cascaded third-harmonic generation in a $2 \mathrm{D}$ nonlinear photonic crystal. Opt. Express. 2017, 25(10), 11556.

17. Wang, Y.; Wei, D. Z.; Zhu, Y. Z.; Huang, X. Y.; Fang, X. Y.; Zhong, W. H.; Wang, Q. J.; Zhang, Y.; Xiao, M. Conversion of the optical orbital angular momentum in a plasmon-assisted second-harmonic generation. Appl. Phys. Lett. 2016. 109(8), 081105.

18. Feng, J.; Cheng, X.; Li, X.; Wang, P.; Hua, W.; Han, K. Highly Efficient Mid-Infrared Generation from Low-Power Single-Frequency Fiber Laser Using Phase-Matched Intracavity Difference Frequency Mixing. Appl. Sci. 2020, 10(21), 7454. https://doi.org/10.3390/app10217454 - 23 Oct 2020

19. Fang, X.; Kuang, Z. Y.; Chen, P.; Yang, H. C.; Li, Q.; Hu, W.; Lu, Y.; Zhang, Y.; Xiao, M. Examining second-harmonic generation of high-order LG modes through a single cylindrical lens. Opt. Lett. 2017, 42(21), 4387.

20. Jingwei Y.; Cheng W.; Efficient terahertz generation scheme in a thin-film lithium niobate-silicon hybrid platform. Optics Express. 2021, 29(11) 16477-16486.

21. Li, Z.; Sun, X.; Zhang, H.; Li, Y.; Yuan, B.; Jiao, B.; Zhao, J.; Tan, L.; Bing, P.; Wang, Z.; Yao, J. High-efficiency terahertz wave generation in aperiodically poled lithium niobate by cascaded difference frequency generation. Journal of the Optical Society of America. 2020, B 37(8), 2416-2422.

22. Carnio, B. N.; Elezzabi, A. Y. Backward terahertz difference frequency generation via modal phase-matching in a planar LiNbO3 waveguide. Optics Letters. 2020, 45(13) 3657-3660.

23. Sato, Y.; Tang, C.; Watanabe, K.; Ohsaki, J.; Yamamoto, T.; Tezuka, N.; Tanabe, T.; Oyama, Y. Terahertz wave generation via difference frequency generation using 2D InxGa1-xSe crystal grown from indium flux. Optics Express. 2020, 28(1) $472-477$.

24. Fang, X; Yang, H; Zhang, Y; Xiao, M; Optical Parametric Amplification Of A Laguerre-Gaussian Mode. OSA Continuum 2019, 2(2), 236-243. [CrossRef]

25. Lanning, R. N.; Xiao, Z.; Zhang, M.; Novikova, I.; Mikhailov, E. E.; Dowling, J. P. Gaussian-beam-propagation theory for nonlinear optics involving an analytical treatment of orbital-angular-momentum transfer. Phys. Rev. 2017, A 96(1), 013830.

26. Suh, Z.-S. Simple Analytical Solution of Čerenkov-Type Terahertz Wave Generation via Difference Frequency Generation in Dielectric Waveguides. Journal of Lightwave Technology. 2019, 37(17) 4236-4243.

27. Dada, A., Leach, J., Buller, G. et al. Experimental high-dimensional two-photon entanglement and violations of generalized Bell inequalities. Nature Phys. 2011, 7, 677-680. https://doi.org/10.1038/nphys1996

28. Ding, D. S.; Zhou, Z. Y.; Shi, B. S.; Guo, G. C. Single-photon-level quantum image memory based on cold atomic ensembles. Nat. Commun. 2013, 4(1), 2527.

29. Miyamoto, K.; Sano, K.; Miyakawa, T.; Niinomi, H.; Toyoda, K.; Vallés, A.; Omatsu, T. Generation of high-quality terahertz OAM mode based on soft-aperture difference frequency generation. Opt. Express. 2019, 27, 31840-31849.

30. Miyamoto, K.; Omatsu, T. Tunable terahertz vortex source based on difference frequency generation JSAP-OSA Joint Symposia. 2019, Paper 19p_E215_2.

31. Qinggang, L.; Shuiqin, Z.; Qiying, S.; Xuanke, Z.; Yi, C.; Ying, L.; Zhenkuan, C.; Lang, Z.; Xinjian, P.; Shixiang, X. Generation of terahertz vortex pulses without any need of manipulation in the terahertz region. Optics Letters. 2019, 44(4), 887-890.

32. Federico, De L.; Cristian, C. Difference-frequency generation in plasmonic nanostructures: a parameter-free hydrodynamic description Journal of the Optical Society of America. 2019, B 36(8) 1979-1986.

33. Jia-Yang, C.; Yong Meng, S.; Zhao-hui, M.; Chao, T.; Zhan, L.; Yu-Ping, H. Efficient parametric frequency conversions in lithium niobate nanophotonic chips Conference on Coherence and Quantum Optics. 2019, Paper W6A.18.

34. Zhang, H; Wang, W.; Jiang, S.; Li, C.; He, Z.; Zhang, S.; Jia, Q.; Wang, L.; He, D. Coherent terahertz radiation with orbital angular momentum by helically microbunched electron beam. AIP Advances. 2021 11, 055115. https://doi.org/10.1063/5.0052083

35. Zhao, Z.; Zhang R.; Song, H. et al. Modal coupling and crosstalk due to turbulence and divergence on free space THz links using multiple orbital angular momentum beams. Sci Rep. 2021;11(1):2110. doi:10.1038/s41598-020-80179-3

36. Meng, M.; Yan, D.; Cao, M.; Li, X.; Qiu, G.; Li, J. Design of negative curvature fiber carrying multiorbital angular momentum modes for terahertz wave transmission, Results in Physics. 2021, Volume 29, , 104766. https://doi.org/10.1016/j.rinp.2021.104766.

37. Pongrac, B.; Đonlagic, D.; Njegovec, M; Gleich, D. THz Signal Generator Using a Single DFB Laser Diode and the Unbalanced Optical Fiber Interferometer. Sensors. 2020, 20(17), 4862. https://doi.org/10.3390/s20174862 
38. Razeghi, L.; Razeghi, M. Recent Advances in Room Temperature, High-Power Terahertz Quantum Cascade Laser Sources Based on Difference-Frequency Generation by Quanyong Photonics. 2016, 3(3), 42. https://doi.org/10.3390/photonics3030042 07 Jul 2016

39. Consolino, L.; Nafa, M.; De Regis, M.; Cappelli, F.; Bartalini, S.; Ito, A.; Hitaka, M.; Dougakiuchi, T.; Edamura, T.; De Natale, P.; Fujita, K. Direct Observation of Terahertz Frequency Comb Generation in Difference-Frequency Quantum Cascade Lasers. Appl. Sci. 2021, 11(4), 1416. https://doi.org/10.3390/app11041416

40. Ding, D. S.; Zhou, Z. Y.; Shi, B. S.; Zhou, X. B.; Guo, G. C. Linear up-conversion of orbital angular momentum. Opt. Lett. 2012, 37(15), 3270.

41. Yariv, A; Yeh, P. Optical Waves in Crystals. New York, Wiley,1984.

42. Boyd, R.W. Nonlinear optics. Academic Press as an imprint of Elsevier, 2008.

43. Soifer, V.A. Diffraction nanophotonics. Ed by Soifer VA. [In Russian]. Moscow, Fizmatlit Publisher, 2011.

44. Kotlyar, V.V; Kovalev, A.A. Vortex laser beams [In Russian]. Samara, Novaya tekhnika Publisher, 2012.

45. Kotlyar, V.V; Kovalev, A.A; Skidanov R.V; Soifer V.A. Rotating elegant beams of Bessel-Gauss [In Russian]. Computer optics 2014, 38(2), 162-170.

46. Vinogradova, I.L; Meshkov, I.K.; Grakhova, E.P.; Sultanov, A.Kh; Bagmanov, V.Kh; Voronkova, A.V.; Gizatullin A.R. Design principles development of secure RoF segment in subterahertz range providing independent optical modulation by radiochannel frequency characteristics and phased antenna array beamsteering parameter [In Russian]. Computer Optics, 2018, 42(5), 786-799. [CrossRef] 